\title{
Grouping variables in an underdetermined system for invariant object recognition
}

\section{Junmei Zhu* and Christoph von der Malsburg}

Address: Frankfurt Institute for Advanced Studies, 60438 Frankfurt am Main, Germany

Email: Junmei Zhu* - jzhu@fias.uni-frankfurt.de

* Corresponding author

from Eighteenth Annual Computational Neuroscience Meeting: CNS*2009

Berlin, Germany. 18-23 July 2009

Published: 13 July 2009

BMC Neuroscience 2009, I0(Suppl I):P308 doi:10.1 I86/I47I-2202-I0-SI-P308

This abstract is available from: http://www.biomedcentral.com/I47I-2202/I0/SI/P308

(c) 2009 Zhu and Malsburg; licensee BioMed Central Ltd.

\section{Introduction}

We study the problem of object recognition invariant to transformations, such as translation, rotation and scale. A system is underdetermined if its degrees of freedom (number of possible transformations and potential objects) exceed the available information (image size). The regularization theory solves this problem by adding constraints [1]. It is unclear what constraints biological systems use. We suggest that rather than seeking constraints, an underdetermined system can make decisions based on available information by grouping its variables. We propose a dynamical system as a minimum system for invariant recognition to demonstrate this strategy.

\section{A dynamical system for invariant recognition} Assume there are $q$ objects in the gallery, and $p$ possible transformations. An input image $I$ is generated by one of the objects through a transformation. The task is to recover the object and the transformation that generate $I$. The system variables are $C=\left(c_{1}, \ldots, c_{p}\right)^{T}$ for transformation and $D=\left(d_{1}, \ldots, d_{q}\right)^{T}$ for object selection. When $p+q>n$, where $n$ is the size of the image, the system is underdetermined, having many solutions.

Our system structure is shown in Figure 1. The state variables $C$ and $D$ follow the dynamics described by a system of linear differential equations. Figure 2 top row shows a solution of a toy system $\left(n=8^{*} 8, p=72, q=2\right)$, with $I$ generated by $c_{1}=1, d_{1}=1$. In this underdetermined system, the solution depends on the initial condition.
The system can be made overdetermined by grouping variables such that all variables within a group share the same dynamics. When the total activity of the system is

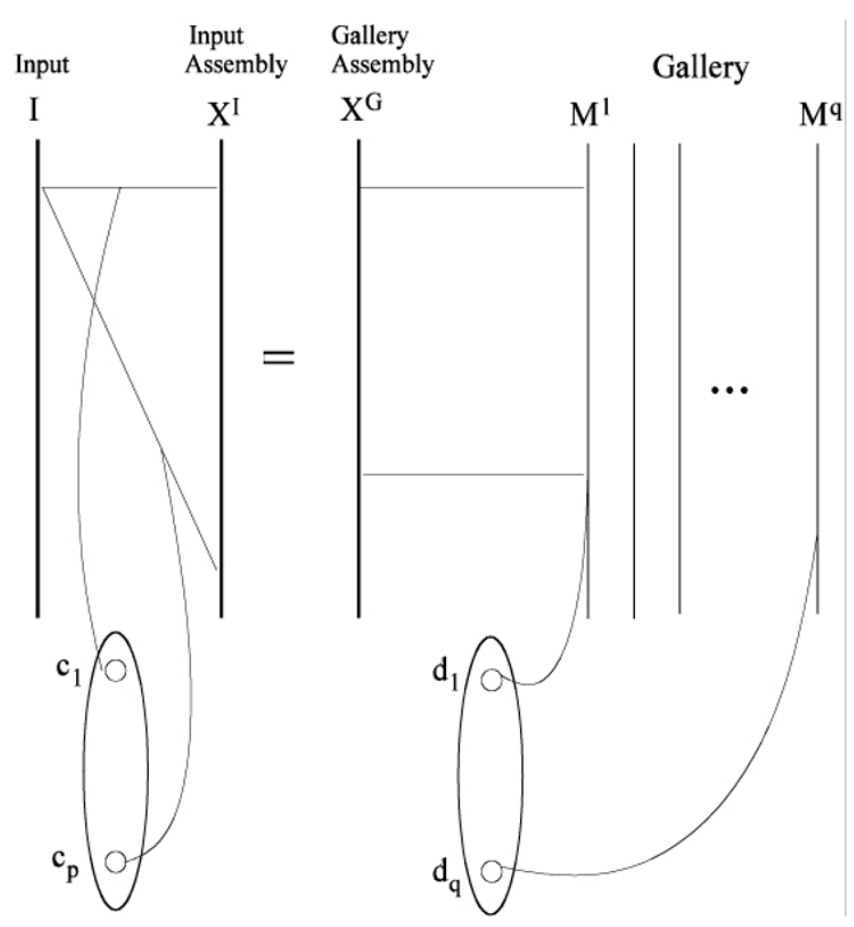

Figure I

System architecture. 

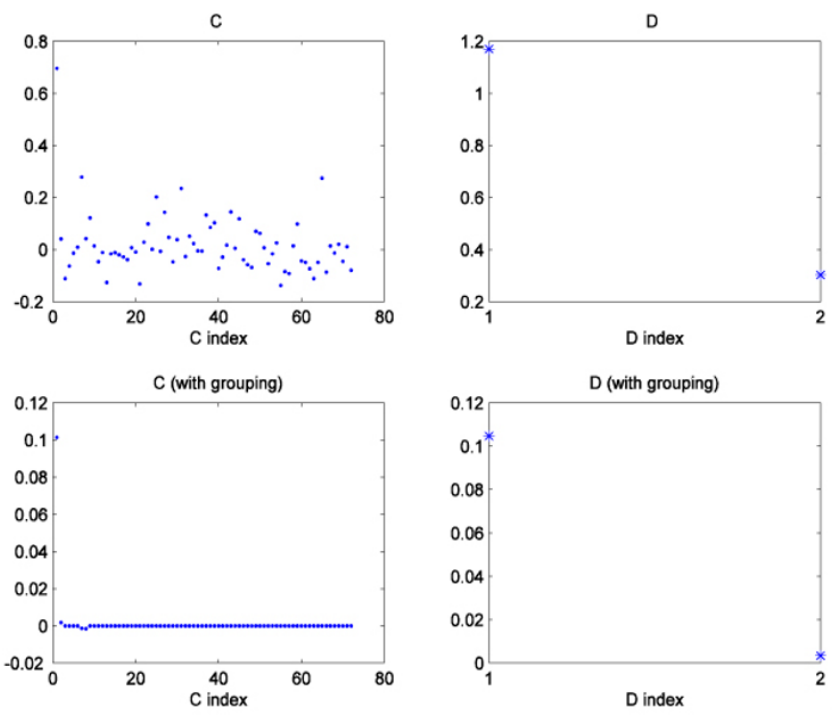

Figure 2

Solutions (C and $D)$ in a toy system.

below a predefined level, we then let the variables in the top group resume their individual dynamics. Under this dynamics with grouping, the solution to the same toy system is shown in Figure 2 bottom row. It is close to the true value.

\section{Discussion}

Our example shows that, in an underdetermined system for invariant recognition, it is plausible to recover a sparse solution by grouping variables and then fine-tune the winning group. The applicability of this strategy depends on the structure of transformations and of objects. Our system could provide a model system to study the coarseto-fine processing which is evident in biological systems [2].

\section{Acknowledgements}

Supported by EU project "SECO" and the Hertie Foundation.

\section{References}

I. Poggio T, Koch C: III-posed problems in early vision: From computational theory to analog networks. Proceedings of the Royal Society London B 1985, 226:303-323.

2. Hegdé J: Time course of visual perception: Coarse-to-fine processing and beyond. Progress in Neurobiology 2008, 84:405-439. 